\title{
Flow Sensing, Estimation and Control for Rotorcraft in Ground Effect
}

\author{
Chin Gian Hooi \\ Graduate Research Assistant \\ hooic@umd.edu \\ Francis D. Lagor \\ Graduate Research Assistant \\ flagor@umd.edu \\ Derek A. Paley \\ Willis H. Young Jr. Associate Professor \\ dpaley@umd.edu \\ Department of Aerospace Engineering and Institute for Systems Research \\ University of Maryland, College Park, MD, USA
}

\begin{abstract}
This paper describes a dynamic controller for rotorcraft landing and hovering in ground effect using feedback control based on flowfield estimation. The rotor downwash in ground effect is represented using a potential flow model selected for real-time use. A nonlinear dynamic model of the rotorcraft in ground effect is presented with open-loop analysis and closedloop control simulation. Flowfield velocity measurements are assimilated into a grid-based recursive Bayesian filter to estimate height above ground. Height tracking in ground effect and landing are implemented with a dynamic linear controller. The overall framework is applicable for rotorcraft operation in ground effect and landing.
\end{abstract}

\section{TABle of Contents}

1 InTRODUCTION $\ldots \ldots \ldots \ldots \ldots \ldots \ldots \ldots \ldots \ldots \ldots, 1$

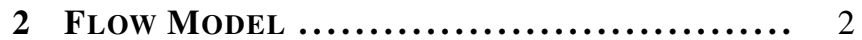

3 DYNAMICS AND CONTROL ................. 4

4 Height and SPEed Estimation ........... 5

5 Conclusion $\ldots \ldots \ldots \ldots \ldots \ldots \ldots \ldots \ldots \ldots \ldots \ldots, 7$

ACKNOWLEDGMENTS ................... 7

REFERENCES $\ldots \ldots \ldots \ldots \ldots \ldots \ldots \ldots \ldots \ldots \ldots \ldots, 7$

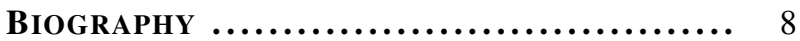

\section{INTRODUCTION}

Rotorcraft operation in ground effect (IGE) presents substantial challenges for vehicle control, including landing with low-impact velocity and maintaining near-ground hover in low-visibility conditions such as brownout [1], fog [2], snow or darkness. Safe operation IGE requires a controller capable of handling uncertainty. Previous authors have developed landing controllers based on robust or adaptive control techniques. For example, Serra and Cunha [3] adopt an affine parameter-dependent model that describes the helicopter linearized error dynamics for a predefined landing region and implements $\mathrm{H}_{2}$ feedback control. Mahony and Hamel [4] develop a parametric adaptive controller that estimates the helicopter aerodynamics onboard and modulates the motor torque, rather than the collective pitch, during takeoff and landing and takes advantage of the reduced sensitivity of the control input to aerodynamics effects. Nonaka and Sugizaki [5] implement ground-effect compensation and integral sliding mode control to suppress the modeling error of the vehicle dynamics in ground effect. These control techniques often require a system model with empirically fit aerodynamic coefficients that are unique to each vehicle.

Safe operation IGE also requires accurate estimation of

978-1-4799-5380-6/15/\$31.00 @ 2015 IEEE. the proximity and relative orientation of the ground plane. Height-estimation methods currently exist for micro aerial vehicles (MAVs) based on ultrasonic, barometric pressure or optical sensors. However, ultrasonic sensors work only for proximity sensing and do not work well for an angled or irregular ground plane [6]. Barometric pressure sensors typically work well for large height differentials [7], but are sensitive to fluctuations in atmospheric pressure, which results in sensor drift. Likewise, the effectiveness of visionbased sensors is limited in degraded visual environments. This paper develops a hover and landing controller that uses rotor downwash flow velocity measurements and an aerodynamic model to estimate the height above ground, thereby providing an additional sensing modality for hovering and landing IGE.

Previous authors have quantified ground effect empirically or through the use of an underlying aerodynamic model. Nonaka and Sugizaki [5] take an empirical approach to measuring the ground effect on rotor thrust as a function of motor voltage. Mahony and Hamel [4] use an approximation of the down-flow velocity ratio based on a piecewise linear approximation of Prouty [8] to estimate rotor-thrust variation IGE. Higher fidelity analytical models include prescribed wake vortex modeling [9] and free vortex modeling [10], which seek to accurately predict the nature of the rotor wake vortices. Cheeseman and Bennett [11] provide a classic analytical model for ground effect, which we adopt for this work, based on aerodynamic modeling using the method of images. The use of an aerodynamic model permits comparison to measurements from sensors such as multi-component differential pressure airspeed sensors [12]. Lagor et al. [13] and DeVries et al. [14] have previously shown that a reducedorder flow model can be rapidly evaluated within a Bayesian filter to perform similar estimation and control tasks in an uncertain flow environment.

This paper develops a dynamic controller for hover and landing IGE based on a flow model for vehicle height estimation. Rotorcraft downwash IGE is modeled using potential flow theory. We extend the model of Cheeseman and Bennett [11] using multiple ring sources; their mirror images create a ground plane. The reduced-order model relates the flowfield velocities to height IGE; it is capable of relatively fast evaluation for control purposes. A nonlinear dynamic model of rotorcraft landing IGE is presented, assuming a rigid rotor of the sort commonly found in MAV rotorcraft [15]. Height estimation of rotorcraft IGE using spatially distributed airspeed measurements is accomplished with a grid-based recursive Bayesian filter. The Bayesian framework is capable of fusing data from additional sensing modalities and for estimation of additional states, such as roll and pitch relative to the landing platform. The feedback controller is implemented in simulation to illustrate the theoretical results. 
The contributions of this paper are (1) a potential-flow model of rotor downwash IGE that more accurately represents the flowfield than previous models; (2) height estimation IGE using multi-component airspeed measurements and a gridbased recursive Bayesian filter; and (3) a flow-estimationbased feedback-control framework for rotorcraft hovering and landing IGE.

The control and estimation architecture developed and simulated in this paper is shown in Fig. 1. The flow velocities $v$ and $w$, in the radial and vertical axes, respectively, are simulated using the potential flow model in Section 2. The rotorcraft dynamics in Section 3 are simulated using a nonlinear state-space model. Flow measurements $\tilde{v}$ and $\tilde{w}$ are presumed to be corrupted with additive sensor noise. Section 4 presents the grid-based recursive Bayesian filter used to estimate the rotorcraft height and a feedback controller that seeks to drive the vehicle to the commanded height.

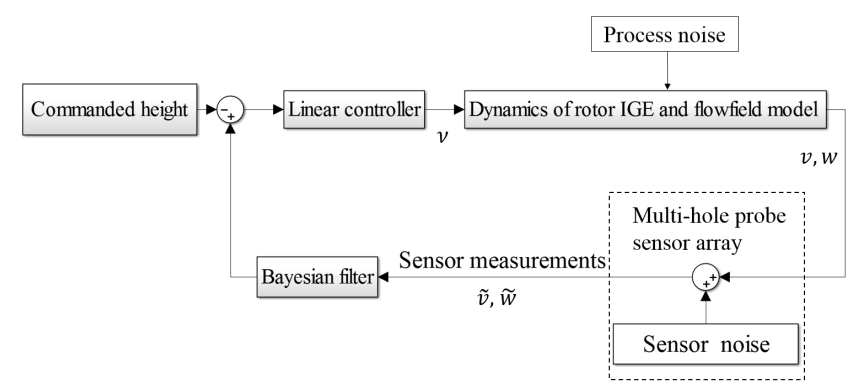

Figure 1. Block diagram for closed-loop flow sensing and control.

\section{FLOW MODEL}

\section{Cheeseman and Bennett Flow Model IGE}

Let $R$ be the rotor radius, $v_{i}$ denote the rotor induced velocity (assume known) and $h$ be the rotor height. Cheeseman and Bennett [11] model the rotor downwash impinging on the ground plane by representing the rotor as a three-dimensional source with strength $s=R^{2} v_{i} / 4$ and the ground plane as a mirror-image source to enforce no flow through the ground plane, as shown in Fig. 2. The sources are separated by a distance $2 h$. The velocity potential for the location $(x, y, z)$ in the flowfield is

$\phi=-\frac{s}{\sqrt{x^{2}+y^{2}+(z-h)^{2}}}-\frac{s}{\sqrt{x^{2}+y^{2}+(z+h)^{2}}}$.

Taking the gradient of the velocity potential with respect to position yields the flow velocity components.

Although the Cheeseman and Bennett flow model has been experimentally shown to accurately capture the relationship between rotor thrust IGE and rotor height[11], it does not accurately represent the physical flowfield of a rotor IGE. As shown in Fig. 2, the flow vectors just below the rotor plane extend radially outward as opposed to downward. Since the rotor is modeled as a point source, the strongest vectors are at the hub and diffuse in strength radially outward.

\section{Ring-Source Potential-Flow Model}

Similar to the Cheeseman and Bennett model, we model the physical flowfield using potential flow theory. However, we expand the single source of Cheeseman and Bennett radially

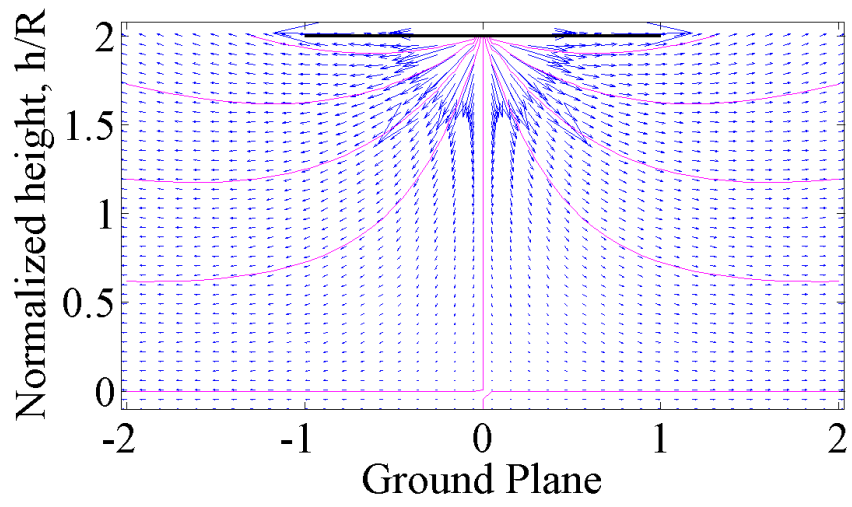

Figure 2. Cheeseman and Bennett [11] potential flow model of rotor downwash in ground effect.

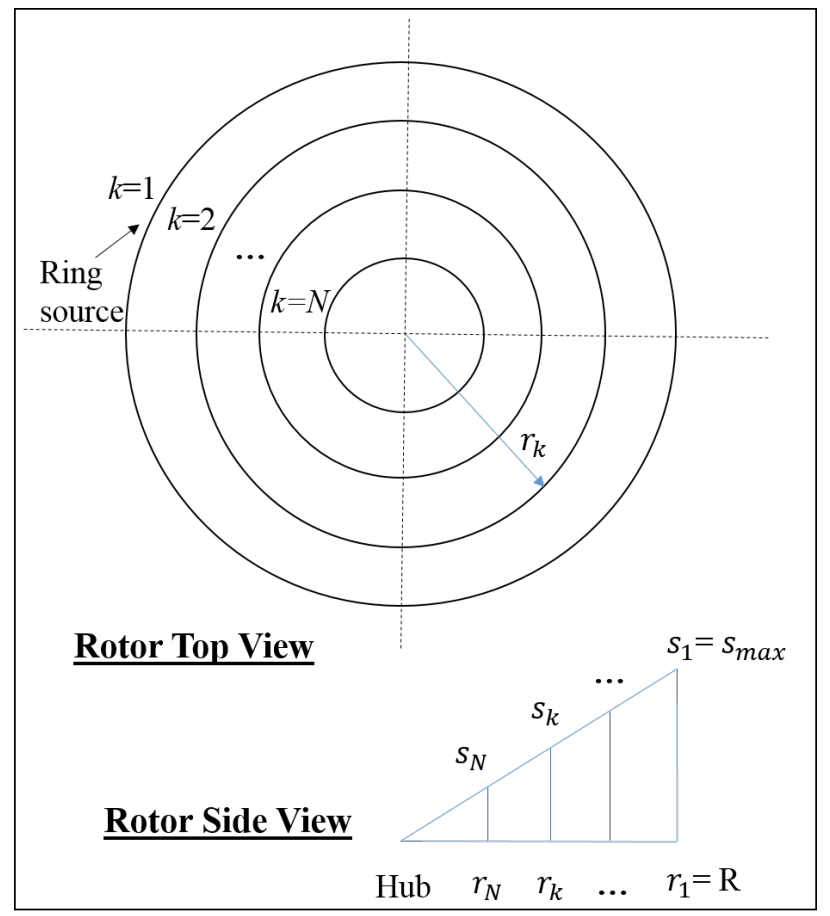

Figure 3. Schematic of ring-source potential-flow model nomenclature.

outward into ring sources to create a more uniform spatial distribution of the flowfield sources.

As shown in Fig. 3, the rotor is modeled by $k=1,2, \ldots, N$ ring sources and the ground plane as their mirror-image ring sources to enforce no flow through the ground plane. Note that ring $k=1$ is at the rotor tip and the ring indices move radially inward with equal radial spacing of $R / N$. The radial location of each ring is

$$
r_{k}=R-(k-1) \frac{R}{N} .
$$

Similar to the inflow ratio distribution of a rotor [16], the strength $s_{k}$ of ring $k$ varies with radial location according to

$$
s_{k}=\frac{s_{\max }}{R} r_{k}
$$

where the maximum source strength $s_{\max }$ is located at the rotor tip $r_{1}=R$. We choose the source strengths according 


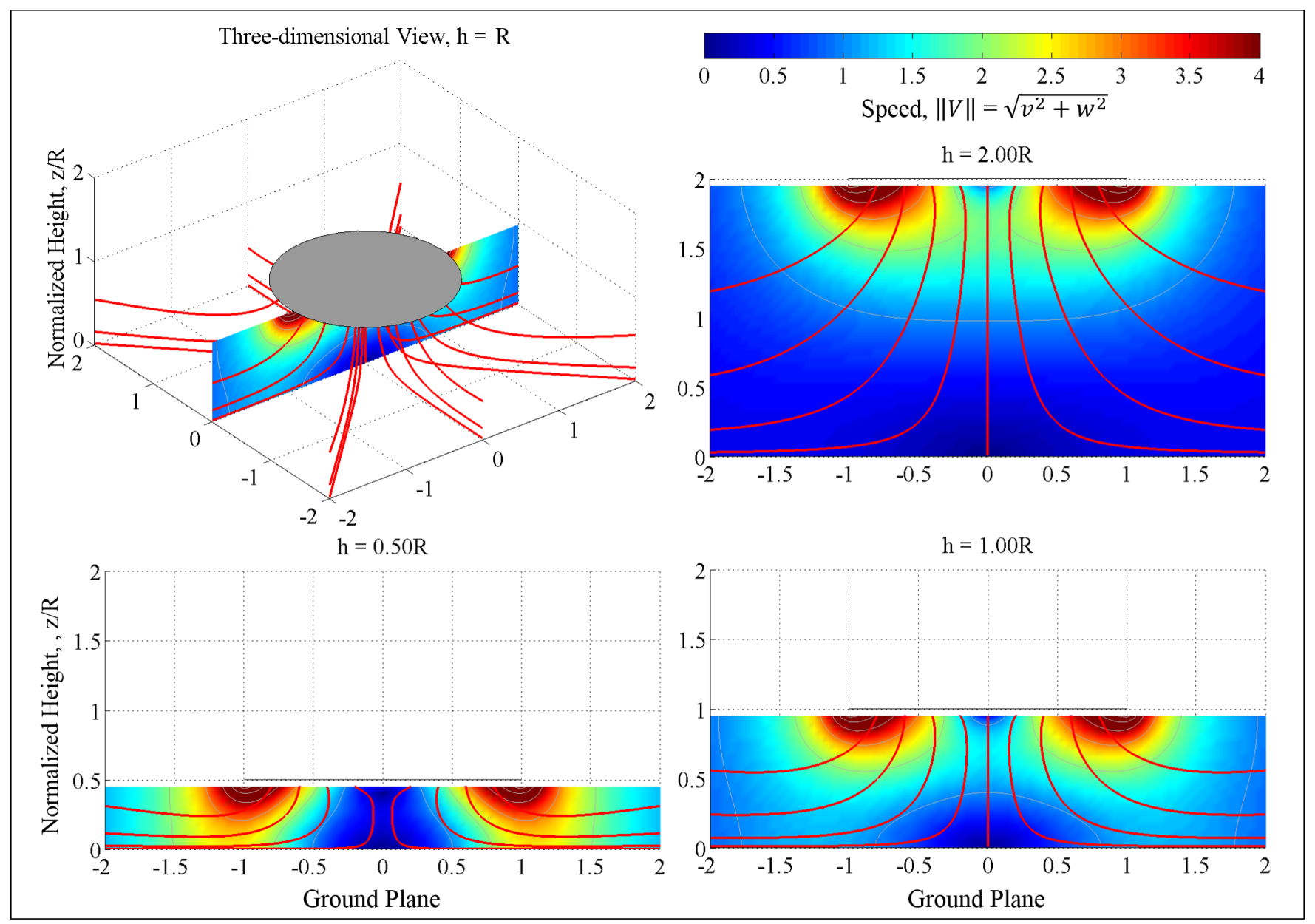

Figure 4. Flowfield of ring-source potential-flow model evaluated at various heights, depicting streamlines and speed distributions, where speed $\|V\|=\sqrt{v^{2}+w^{2}}$.

to the total volumetric flow through the rotor disk, similar to Cheeseman and Bennett. Let $A=\pi R^{2}$ denote the rotor disk area. The strength of each ring source represents the volumetric flow rate per unit length and the total flow rate satisfies

$$
\sum_{k=1}^{N} 2 \pi r_{k} s_{k}=A v_{i}
$$

Substituting (2) and (3) into (4) and using arithmetic series along with the sum of a sequence of squares yields

$$
s_{\max }=\frac{3 N A v_{i}}{\pi R\left(2 N^{2}+3 N+1\right)}=\frac{3 N R v_{i}}{\left(2 N^{2}+3 N+1\right)} .
$$

Note that, for $N=1$,

$$
s_{\max }=\frac{A v_{i}}{2 \pi R}=\frac{R v_{i}}{2} .
$$

The velocity potential of ring source $k$ is [17]

$$
\phi_{k}\left(r, z ; r_{k} z_{k}\right)=\frac{-s_{k} r_{k} K(M)}{\pi \sqrt{\rho_{1}\left(r, z ; r_{k} z_{k}\right)}},
$$

where $\rho_{1}=\left(r+r_{k}\right)^{2}+(z-h)^{2}, r$ and $z$ are the radial location and elevation of the query point, respectively, and $M=4 r r_{k} / \rho_{1}$. The radial $v_{k}\left(\rho_{1}, \rho_{2}\right)$ and vertical $w_{k}\left(\rho_{1}, \rho_{2}\right)$ velocity components of ring source $k$ are [17]

$$
\begin{gathered}
v_{k}=\frac{r_{k} s_{k}}{2 \pi r \sqrt{\rho_{1}}}\left[K(M)+\frac{r^{2}-r_{k}^{2}-(z-h)^{2}}{\rho_{2}} E(M)\right] \\
\text { and } \quad w_{k}=\frac{s_{k} r_{k}(z-h) E(M)}{\pi \rho_{2} \sqrt{\rho_{1}}}
\end{gathered}
$$

where $\rho_{2}=\left(r-r_{k}\right)^{2}+(z-h)^{2}$ and $K(M)$ and $E(M)$ are the first and second complete elliptic integrals respectively. $(K(M)$ and $E(M)$ are evaluated using the ellipke function in MATLAB ${ }^{\circledR}$.) The velocity components of the flowfield are the sum of each ring source and their image ring source contributions,

$$
\begin{array}{r}
v(r, z)=\sum_{k=1}^{N} v_{k}\left(\rho_{1}, \rho_{2}\right)+\sum_{k=1}^{N} v_{k}\left(\overline{\rho_{1}}, \overline{\rho_{2}}\right), \\
w(r, z)=\sum_{k=1}^{N} w_{k}\left(\rho_{1}, \rho_{2}\right)+\sum_{k=1}^{N} w_{k}\left(\overline{\rho_{1}}, \overline{\rho_{2}}\right),
\end{array}
$$

where $\overline{\rho_{1}}=\left(r+r_{k}\right)^{2}+(z+h)^{2}$ and $\overline{\rho_{2}}=\left(r-r_{k}\right)^{2}+(z+h)^{2}$. 
Fig. 4 shows the flowfield simulation of the ring-source potential-flow model, with streamlines and speed distribution shown for different heights, where speed $\|V\|=\sqrt{v^{2}+w^{2}}$. These variations in speed distribution with height serve as an informative tool for sensor placement to measure the physical flowfield. This potential flow model is qualitatively similar to the flow visualization model of the flow below a rotor IGE by Lee et al. [18].

Moving from the rotor plane to the ground close to the rotor hub, the flow decelerates and forms a stagnation region close to the ground. The flow deceleration region is easiest to distinguish for $h=1.00 \mathrm{R}$ in Fig. 4. In contrast, the flow acceleration region is where the streamlines change direction from pointing downward to radially outward. As the rotor approaches the ground, the streamlines are compressed, which is best illustrated for $\mathrm{h}=0.50 \mathrm{R}$ in Fig. 4. Evidently, the flow speed is the highest in the flow acceleration region for the $\mathrm{h}=0.50 \mathrm{R}$ case as opposed to the $\mathrm{h}=2.00 \mathrm{R}$ case, since the flow is being compressed more with less space between the rotor plane and the ground. This effect is analogous to moving a water jet (the rotor) closer to a wall (the ground plane), where the jet speed in the flow acceleration region is highest when it is closest to the wall.

Although the rotor downwash IGE is by no means laminar, as visualized in the work of Lee et al. [18], we can model it using potential flow theory and account for turbulence with process noise (to be introduced in Section 4). Effectively, we model the mean velocity of the dominant flow and treat the turbulence and other secondary effects such as blade tip vortices as fluctuations away from this mean.

Suppose differential pressure airspeed sensor measurements, $\tilde{V}=[\tilde{v}, \tilde{w}]^{T}$ are collected below the rotor in the experimental setup. Airspeed measurements of the sort described in [12] contain two components of the flow velocity at each sensor location and are collected in an array configuration to probe the flowfield at multiple spatial locations. We assume measurements $\tilde{V}$ of the flow velocities are corrupted by zeromean Gaussian white noise $\eta$ with standard deviation $\sigma_{\eta}$ and zero mean, resulting in the measurement model

$$
\tilde{V}=V+\eta
$$

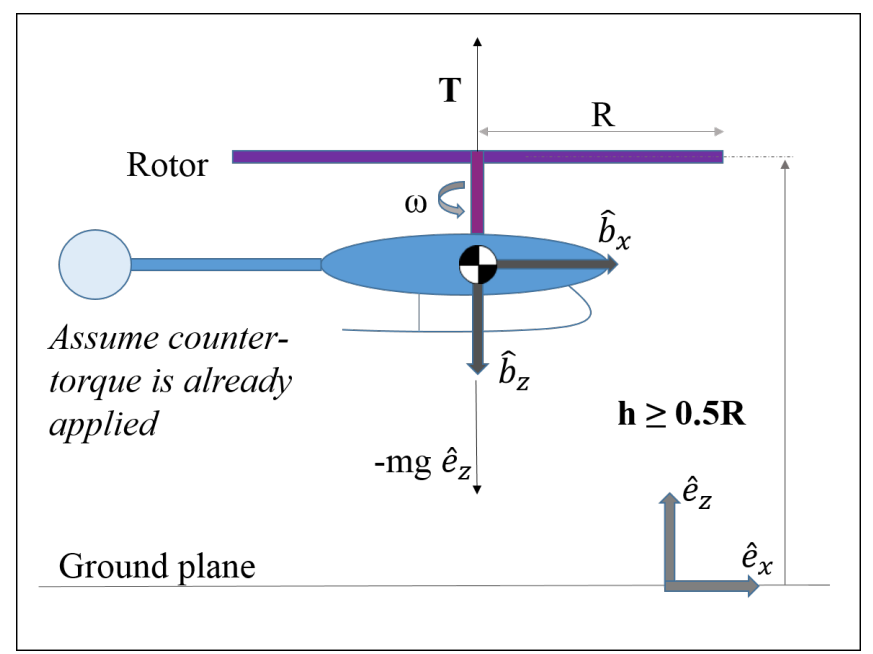

Figure 5. Free-body diagram of rotorcraft in ground effect.

\section{DYNAMICS AND CONTROL}

\section{Dynamics of Rotorcraft Operation in Ground Effect}

Fig. 5 shows the free-body diagram of a rotorcraft in which the tailrotor counter torque is not shown. Applying Newton's second law in the $z$-direction yields

$$
m \ddot{h}=T-m g
$$

where $\ddot{h}$ is the vertical acceleration. Modeling the rotor thrust $T$ as a function of rotor rotational speed $\omega$ yields [15]

$$
T=k \omega^{2} \text {. }
$$

The rotor thrust is augmented for ground effect using the Cheeseman and Bennett model, which captures the essential characteristic of the relationship between thrust and height IGE,

$$
T_{I G E}=\frac{1}{1-\frac{R^{2}}{16 h^{2}}} T=\frac{16 h^{2}}{16 h^{2}-R^{2}} T
$$

Based on experimental data, Leishman [16] suggests that model (16) is valid for $h / R \geq 0.5$. It is assumed henceforth that the rotorcraft has landed at the boundary condition of $h / R=0.5$, which is reasonable since the rotor distance above the landing gear is generally greater than $0.5 R$. Thrust IGE is substituted into the dynamics in (14) to obtain

$$
\ddot{h}=\frac{16 h^{2} k \omega^{2}}{\left(16 h^{2}-R^{2}\right) m}-g .
$$

The state vector $Z \in \mathbb{R}^{2}$ is defined as

$$
Z=\left[\begin{array}{l}
h \\
\dot{h}
\end{array}\right]=\left[\begin{array}{l}
z_{1} \\
z_{2}
\end{array}\right],
$$

where $\dot{h}$ is the landing speed. Since the rotor rotational speed is regulated, we define $\nu=k \omega^{2} / m$ as the control input. The nonlinear state space form is

$$
\dot{Z}=\left[\begin{array}{l}
\dot{h} \\
\ddot{h}
\end{array}\right]=\left[\begin{array}{c}
z_{2} \\
\frac{16 z_{1}^{2}}{16 z_{1}^{2}-R^{2}} \nu-g
\end{array}\right] .
$$

An equilibrium control input $\nu^{*}$ is necessary to keep the rotorcraft hovering at a corresponding equilibrium height $z_{1}^{*}$ (or to land safely). Solving (19) for the equilibrium condition, $\dot{Z}^{*}=0$, the equilibrium control input is

$$
\nu^{*}=g \frac{16 z_{1}^{* 2}-R^{2}}{16 z_{1}^{* 2}} .
$$

Fig. 6 depicts the simulation results of the open-loop nonlinear dynamics for initial height and speed $(1.5 \mathrm{~m}$ and $0.25 \mathrm{~m} / \mathrm{s})$ and constant input $\nu=\nu^{*}$.

In order to implement a linear controller for the nonlinear dynamics (19), the Jacobian matrices are needed. The Jacobians

$$
A=\left[\begin{array}{cc}
0 & 1 \\
\frac{-2 g R^{2}}{z_{1}^{*}\left(16 z_{1}^{* 2}-R^{2}\right)} & 0
\end{array}\right] \quad \text { and } \quad B=\left[\begin{array}{c}
0 \\
\frac{16 z_{1}^{* 2}}{16 z_{1}^{* 2}-R^{2}}
\end{array}\right]
$$

are the partial derivatives of the right-hand side of (19) with respect to $Z$ and $\nu$, respectively. The linear system dynamics are

$$
\dot{Z}=A Z+B \nu
$$




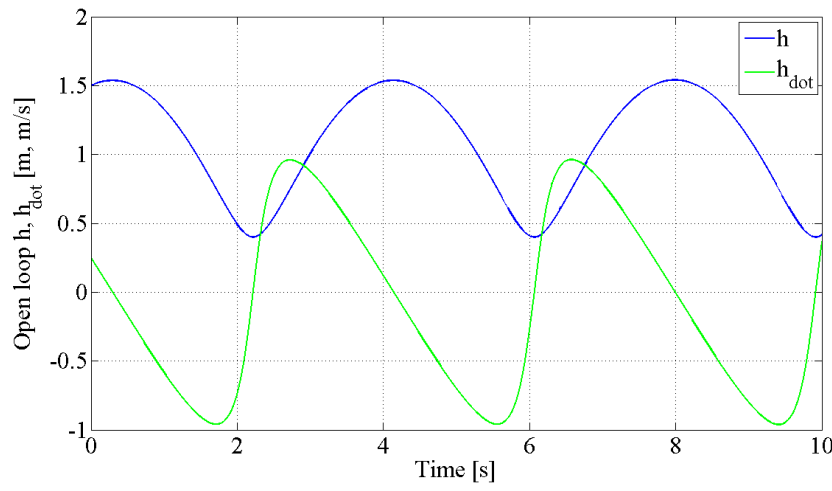

Figure 6. Open-loop dynamics of rotorcraft in ground effect with constant input $\nu=\nu^{*}$. Initial conditions for height and speed are $(1.5 \mathrm{~m}, 0.25 \mathrm{~m} / \mathrm{s})$.

\section{Nonlinear Dynamics with Linear Observer-based Feedback Control}

The state space system (19) in control affine form is

$$
\dot{Z}=f(Z)+g(Z) \nu,
$$

where

$$
f(Z)=\left[\begin{array}{c}
z_{2} \\
-g
\end{array}\right] \text { and } g(Z)=\left[\begin{array}{c}
0 \\
\frac{16 z_{1}^{2}}{16 z_{1}^{2}-R^{2}}
\end{array}\right] .
$$

Fig. 6 shows that the constant-input open-loop nonlinear system with $\nu=\nu^{*}$ oscillates about the equilibirum point, which implies that feedback control is needed to asymptotically stabilize $z_{1}$ to the desired height. We design a linear controller to be used with the nonlinear system dynamics,

$$
\nu=\nu^{*}+\Delta \nu,
$$

where $\Delta \nu=-K\left(\hat{Z}-Z^{*}\right), \quad K=\left[\begin{array}{ll}K_{1} & K_{2}\end{array}\right]$ and $\hat{Z}=\left[\begin{array}{ll}\hat{z}_{1} & \hat{z}_{2}\end{array}\right]^{T}$ denotes the estimated states. The closed-loop dynamics with the linear output-feedback controller (25) are

$$
\dot{Z}=\left[\begin{array}{c}
z_{2} \\
-g
\end{array}\right]+\left[\begin{array}{c}
0 \\
\frac{16 z_{1}^{2}}{16 z_{1}^{2}-R^{2}}
\end{array}\right]\left(\nu^{*}+\Delta \nu\right),
$$

i.e.,

$\dot{Z}=\left[-g+\frac{16 z_{1}^{2}}{16 z_{1}^{2}-R^{2}}\left(g \frac{16 z_{1}^{* 2}-R^{2}}{16 z_{1}^{* 2}}-K_{1}\left(\hat{z}_{1}-z_{1}^{*}\right)-K_{2} \hat{z}_{2}\right)\right]$.

Figure 7 shows the nonlinear closed-loop dynamics (27) and the linear dynamics (22) with the linear controller (25). The simulation is implemented using full-state feedback, $\hat{Z}=Z$. The optimal gains $K$ are provided by a Linear Quadratic Regulator (LQR) and the Jacobian matrices in (21) evaluated at the equlibrium height. Initial conditions for the height and speed are $(1.8 \mathrm{~m}, 0.9 \mathrm{~m} / \mathrm{s})$ and desired steady-state conditions are $(0.75 \mathrm{~m}, 0 \mathrm{~m} / \mathrm{s})$.

\section{Height and Speed Estimation}

The Bayesian filter [14] [19] is a probabilistic approach for estimation that assimilates noisy measurements into a probability density function (PDF) and also allows the incorporation of nonlinear system dynamics and nonlinear observation

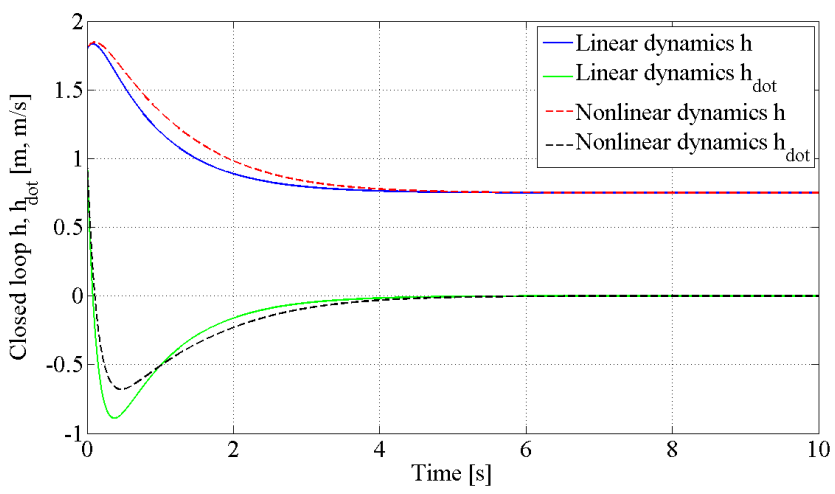

Figure 7. Closed-loop dynamics of rotorcraft in ground effect with full-state feedback, $\hat{Z}=Z$ using the linear controller (25).

operators. (The optimal Bayesian filter for linear systems with linear measurements and Gaussian noise is the Kalman filter [20], while a common Bayesian filter for nonlinear systems with nonlinear observation and noise model is the particle filter [21].) We chose a grid-based recursive Bayesian filter as it can be rapidly implemented for a low-dimensional state-space representation of the rotorcraft downwash with linear parameter estimates and nonlinear measurement models. ${ }^{2}$ It is of note that even though linear paramater estimates and Gaussian white noise is assumed for our measurement and process noise, these are not required assumptions for the Bayesian filter.

\section{Estimation Step}

The Bayesian framework consists of estimation and prediction steps. In the estimation step, the Bayesian filter in the form of [14] is used to estimate the vehicle height based on the flow velocity measurements from an array of differential pressure airspeed sensors. Grid-based Bayesian estimation is performed recursively, in which the finite parameter space over height $h$ is discretized and the PDFs are evaluated on this grid for each new measurement. Let $h$ be the single state of a one-dimensional Bayesian filter. Recall that the noisy flow measurement $\tilde{V}$ is corrupted with zero mean Gaussian noise as in (13). Let $L=\left\{\tilde{V}_{1}, \ldots, \tilde{V}_{m}\right\}$ denote the set of observations from $m$ sensors. The posterior probability of the state $h$ given the measurements $L$ is [14]

$$
P(h \mid L)=c P(L \mid h) P\left(h \mid L_{0}\right),
$$

where $\mathrm{c}$ is the scaling factor chosen so that $P(\hat{h} \mid L)$ has unit integral over the state space. The likelihood function $P(L \mid h)$ is the conditional probability of the observations $L$ given the state $h$ and $P\left(h \mid L_{0}\right)$ represents the prior probability distribution. During initialization or in the absence of measurements, the prior probability $P\left(h \mid L_{0}\right)$ is uniform.

We choose a Gaussian likelihood function for the measurements $\tilde{V}_{l}, l=1, \ldots, m$, i.e.

$$
P\left(\tilde{V}_{l} \mid \hat{h}\right)=\frac{1}{\sqrt{2 \pi} \sigma} \exp \left[-\frac{1}{2 \sigma^{2}}\left\|\tilde{V}_{l}-V_{l}\right\|^{2}\right],
$$

\footnotetext{
${ }^{2}$ The Unscented Kalman filter [22] is an approximate nonlinear estimator that differs the inevitable divergence with highly nonlinear systems or measurements [20]. The particle filter [21] provides high performance estimation but it requires careful selection of its estimation state vector because it is prone to sample impoverishment and requires careful tuning
} 

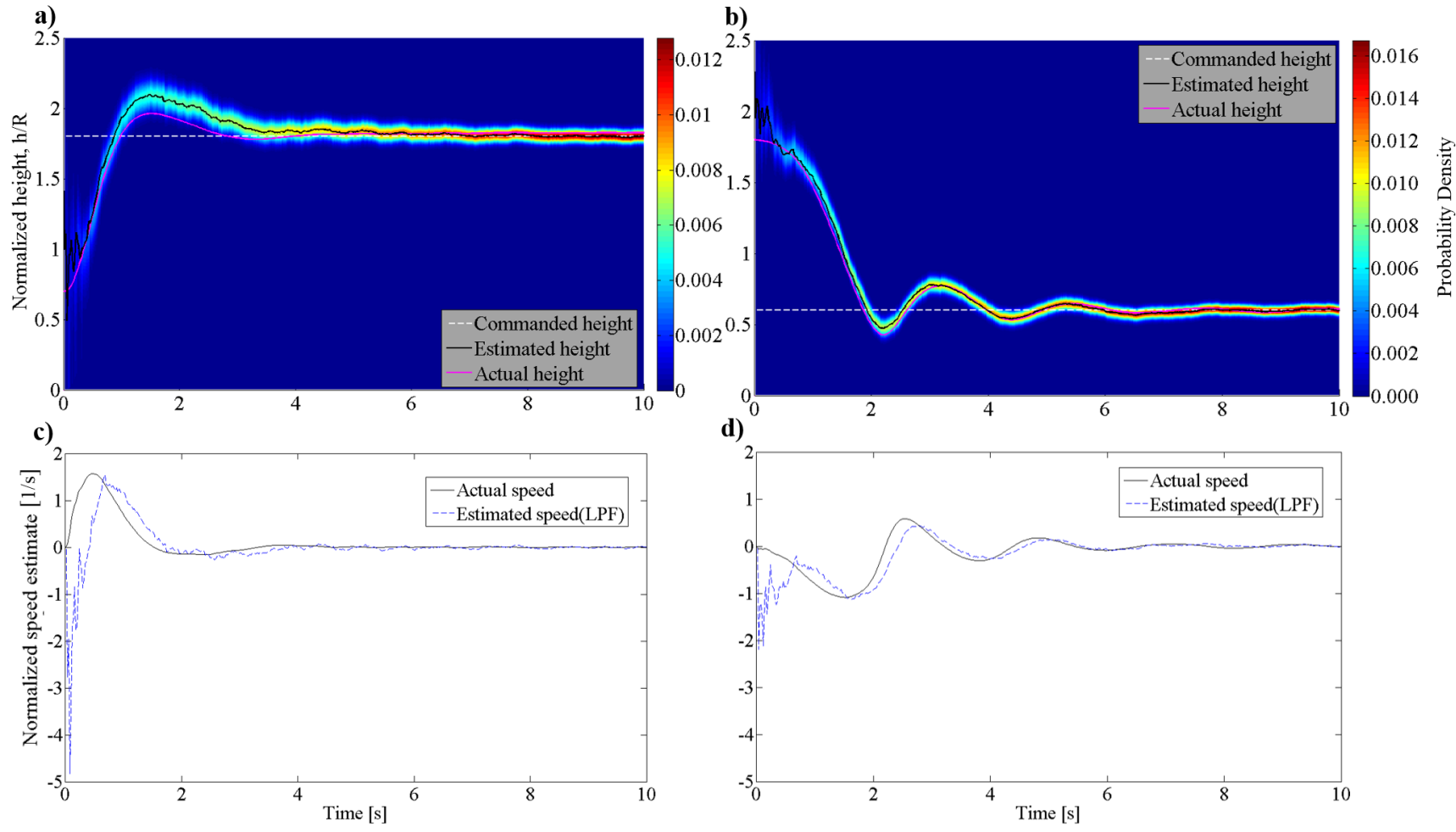

d)

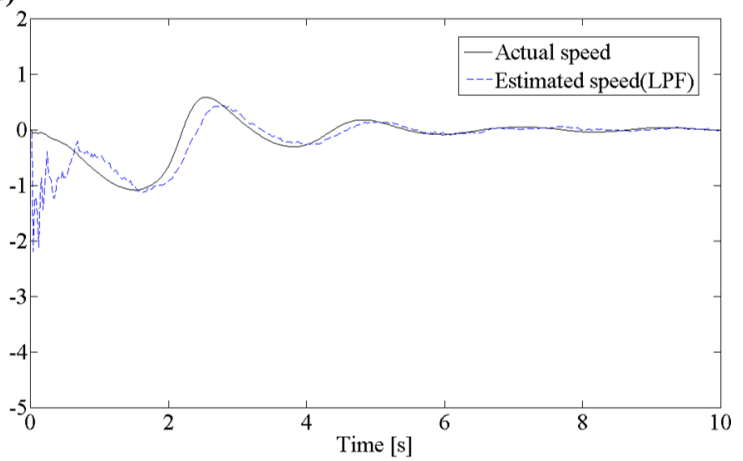

Figure 8. Closed-loop control with estimated height using Bayesian filter framework shows the marginal probability density of normalized height $h / R$ and normalized speed plotted versus time. (a) Ascent maneuver from initial height and speed of ( 0.7 , $0 / \mathrm{s})$, commanded height of 1.8 and process and measurement noise standard deviation of $(0.1,0.15 / \mathrm{s})$. (b) Descent maneuver from initial height and speed of $(1.8,0.2 / \mathrm{s})$, commanded height of 0.6 and process measurement noise standard deviation of $(0.08,0.1 / \mathrm{s})$. (c) Estimated speed using low-pass-filtered (LPF) finite differencing for ascent maneuver in (a). (d) Estimated speed using low-pass-filtered (LPF) finite differencing for descent maneuver in (b).

where $V_{l}$ is generated from the flow models (11) and (12) at sensor location $l$ embedded within the Bayesian filter and $\sigma^{2}$ is the measurement variance. Even though (29) is of the Gaussian form, it is not strictly required. The posterior probability density of the state $h$ is obtained using the joint measurement likelihood combining the measurements taken from $m$ sensors [14]

$$
P(h \mid L)=c\left(\prod_{l=1}^{m} P\left(\tilde{V}_{l} \mid h\right)\right) P\left(h \mid L_{0}\right) .
$$

The estimated height $\hat{h}$ corresponding to the mode (maximum) of the posterior probability $P(h \mid L)$ provides the maximum likelihood estimate of the flowfield parameters.

Spatial integration over the sensor array is accomplished by (30), whereas temporal integration is accomplished by assigning the posterior of the current time step to be the prior for the next time step.

\section{Prediction Step}

The prediction step consists of shifting and diffusing the probability mass to account for the vehicle dynamics. This is accomplished using the Chapman-Kolmogorov equation [21],

$$
\begin{aligned}
& P(h(t+\Delta t) \mid L(t)) \\
& =\int P(h(t+\Delta t) \mid h(t)) P(h(t) \mid L(t)) d h(t),
\end{aligned}
$$

where $t$ is the current time step and $\Delta t$ is the time step interval.

Numerically, the probability density is shifted along the grid according to the estimated speed $\hat{z}_{2}$. If the estimated speed $\hat{z}_{2}$ is positive, we shift the PDF to the right and vice-versa. The number of grid points to shift is determined by the multiplication of the estimated speed $\hat{z}_{2}$ and simulation time interval. After shifting, the probability density is normalized to ensure the sum of the area under the PDF equals to one.

The probability density is diffused with process noise $\gamma$ by convolution with a grid-sized Gaussian window whose width is inversely proportional to the standard deviation of the process noise $\sigma_{\gamma}$. (This is done with the MATLAB ${ }^{\circledR}$ functions gausswin and convn.)

\section{Simulation Examples}

Fig. 8 shows the evolution of the estimated height marginal probability density for closed-loop ascent in Fig. 8(a) and descent in Fig. 8(b). Fig. 8(a) shows an ascent maneuver from initial normalized height and speed of $(0.7,0 / \mathrm{s})$ to a commanded height of 1.8 and process and measurement noise standard deviation of $(0.1,0.15 / \mathrm{s})$. Fig. 8(b) shows a descent maneuver from initial height and speed of $(1.8,0.2 / \mathrm{s})$ to a commanded height of 0.6 and process and measurement noise standard deviation of $(0.08,0.1 / \mathrm{s})$. Fig $8(\mathrm{c})$ and $(\mathrm{d})$ show the estimated speeds using the low-pass-filtered finitedifferencing method (32) for ascent in (a) and descent in (b), respectively. 
These manuevers are simulated using the closed-loop dynamics (27). The estimated height $\hat{z}_{1}$ is evaluated recursively by the one-dimensional Bayesian filter. The estimated height $\hat{z}_{2}$ is evaluated by finite differencing, i.e.,

$$
\hat{z}_{2, p}=\alpha \hat{z}_{2, p-1}+(1-\alpha) \frac{\hat{z}_{1, p}-\hat{z}_{1, p-1}}{\Delta t}
$$

where $0<\alpha<1$, the index $\mathrm{p}$ indicates the current time step and $\Delta t$ is the time interval between each simulation step. Eq. (32) is a low-pass-filter that removes the effects of noise. Process noise $\gamma$, which is Gaussian white noise with standard deviation $\sigma_{\gamma}$ and zero mean, is added to (27) in the filter.

Initially, Fig. 8(a) and (b) show that the estimated height error is large because the initial prior PDF is uniformly distributed. As time progresses and the Bayesian filter assimilates measurements, the marginal probability density begins to peak and the estimated height converges to the actual height. As more measurements are taken, the filter becomes more confident of its estimates as shown by the narrowing probability density. It is also of note that Fig. 8(a) has a bigger spread throughout its probability density distribution than that of Fig. 8(b) due to the higher noise variances.

Fig. 8(c) and (d) show that the initial speed estimates are relatively large as the difference between succesive height estimations is also relatively large. This is influenced by the Bayesian filter initiation and also the controller which is driving the system to the comanded height. As the system reaches steady-state at about $4 \mathrm{~s}$, the speed estimates begin to closely track the actual speed. The first-order speed estimation could be improved by using a higher-order estimation method.

\section{Conclusion}

This paper describes a dynamic controller for rotorcraft landing and hovering in ground effect. A ring-source flow model for the rotor downwash IGE is developed using potential flow theory that captures the essential characteristics of the relationship between flow velocities and height. The reducedorder flow model is used for fast evaluation of the flowfield in a recursive control loop and still resembles the physical flowfield. A nonlinear dynamic model of rotorcraft landing IGE is also developed using potential flow theory, which allows for the study of the open-loop dynamics and facilitates the design of a closed-loop controller. The height of the rotorcraft IGE can be estimated with a grid-based recursive Bayesian filter using the three-dimensional flow model, nonlinear dynamic model and velocity measurements. Finally, flow-estimation-based closed-loop control is implemented in simulation, which does not require any sensor measurements other than the flow sensors. Experimental validation of the flow estimation and feedback control is ongoing. Additional theoretical and modeling work includes sensor fusion of multiple sensing modalities, such as ultrasonic sensors. The potential flow model and dynamics are being improved to incorporate changes in rotorcraft pitch angle, a moving landing platform, and external disturbances such as wind gusts during landing.

\section{ACKNOWLEDGMENTS}

The authors gratefully thank Daigo Shishika, Nitin Sydney, and Derrick Yeo for valuable discussions related to this work. This work is supported by the University of Maryland Vertical Lift Rotorcraft Center Of Excellence Army Grant
No. W911W61120012.

\section{REFERENCES}

[1] Tritschler, J., "Contributions to the Characterization and Mitigation of Rotorcraft Brownout," Doctor of Philosophy Dissertation, Department of Aerospace Engineering, University of Maryland, 2012.

[2] Plank, V. G., Spatola, A. A., Hicks, J. R., "Fog Modification by Use of Helicopters," Air Force Cambridge Research Laboratories, U.S. Army Atmospheric Sciences Laboratory, AFCRL-70-0593, Environmental Research Papers No. 335, ECOM-5339, October 28, 1970.

[3] Serra, P., Cunha, R., Silvestre, C., "On the Design of Rotorcraft Landing Controllers," 16th Mediterranean Conference on Control and Automation Ajaccio, France, June 25-27, 2008.

[4] Mahony, R., Hamel, T., "Adaptive Compensation of Aerodynamic Effects during Takeoff and Landing Manoeuvres for a Scale Model Autonomous Helicopter,' European Journal of Control, (2001)0:1-15, 2001.

[5] Nonaka, K., Sugizaki, H., "Integral Sliding Mode Altitude Control for a Small Model Helicopter with Ground Effect Compensation," American Control Conference, San Francisco, CA, June 29 - July 01, 2011.

[6] Borenstein, J., Koren, Y., "Obstacle Avoidance with Ultrasonic Sensors," IEEE Journal for Robotics and Automation, Vol. 4, No. 2, April 1988.

[7] Tanigawa, M., Luinge, H., Schipper, L., Slycke, P., "Drift-Free Dynamic Height Sensor using MEMS IMU Aided by MEMS Pressure Sensor," Proceedings of the 5th Workshop on Positioning, Navigation and Communication, 2008.

[8] Prouty, R. W., Helicopter Performance, Stability, and Control, Krieger Publishing Company, Florida, 2002.

[9] Gilad, M., Chopra, I., Rand, O., "Performance Evaluation of a Flexible Rotor in Extreme Ground Effect," 37th European Rotorcraft Forum, Milan, Italy, 2011.

[10] Govindarajan, B., "Evaluation of Particle Clustering Algorithms in the Prediction of Brownout Dust Clouds," Master's Thesis, Department of Aerospace Engineering, University of Maryland, August 2011

[11] Cheeseman, I. C., Bennett, W. E., "The Effect of the Ground on a Helicopter Rotor in Forward Flight," Reports \& Memoranda No. 3021, Ministry of Supply, Aeronautical Research Council Reports and Memoranda, September 1955.

[12] Yeo, D. W., Sydney, N., Paley, D., Sofge, D., "Onboard Flow Sensing For Downwash Detection and Avoidance On Small Quadrotor Helicopters," AIAA Guidance, Navigation and Control Conference 2015, accepted for publication.

[13] Lagor, F. D., DeVries, L. D., Waychoff, K., Paley, D. A., "Bio-inspired Flow Sensing and Control: Autonomous Rheotaxis Using Distributed Pressure Measurements," Journal of Unmanned System Technology, Nov 2013.

[14] DeVries, L. D., Lagor, F. D., Lei, H., Tan, X. ,Paley, D. A., "Distributed Flow Estimation and Closed-Loop Control of an Underwater Vehicle with a Multi-Modal Artificial Lateral Line," Bioinspiration \& Biomimetics, special issue on "Hybrid and Multi-modal Locomotion," accepted for publication. 
[15] Hooi, C. G., "Design, Rapid Prototyping and Testing of a Ducted Fan Microscale Quadcopter," 70th American Helicopter Society Society Annual Forum \& Technology Display, Montreal, Quebec, Canada, May 2014.

[16] Leishman, J. G., Principles of Helicopter Aerodynamics, 2nd. Ed., p.258 - 260, Cambridge University Press, New York, 2006.

[17] Hess, J.L., Smith, A.M.O., "Calculation of Potential Flow about Arbitrary Bodies," Progress in Aerospace Sciences, Volume 8, p. 3940, 1967.

[18] Lee, Ramasamy, Leishman, J. G., "Fluid Dynamics of Interacting Blade Tip Vortices with a Ground Plane," Journal of the American Helicopter Society 55, 022005 , 2010.

[19] Murphy, K., Machine Learning: A Probabilistic Perspective, The MIT Press, Cambridge, Massachusetts, London, 2012.

[20] Simon, D., Optimal State Estimation: Kalman, H Infinity, and Nonlinear Approaches , 1st. Ed., p.465-466, John Wiley \& Sons, Inc., New Jersey, 2006.

[21] Arulampalam, M. S., Maskell, S., Gordon, N., Clapp, T., "A Tutorial on Particle Filters for Online Nonlinear/Non-Gaussian Bayesian Tracking," IEEE Transactions on Signal Processing, Vol. 50, No. 2, February 2002.

[22] Julier, S., Uhlmann, J., "Unscented Filtering and Nonlinear Estimation", Proceedings of the IEEE, Vol. 92, No. 3, March 2004.

\section{BIOGRAPHY}

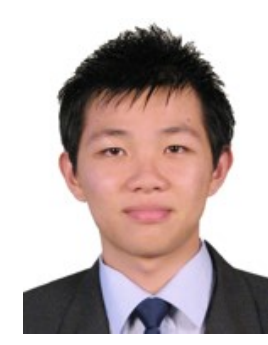

Chin Gian Hooi received the B.S. degree in Aerospace Engineering from Embry-Riddle Aeronautical University in 2013 and is pursuing the M.S. degree in Aerospace Engineering at the University of Maryland. He founded an Unmanned Aerial Vehicle startup, Xronz, before grad school and is involved in design and control. Chin Gian was a 20132014 Tau Beta Pi Fellow and received the Vertical Flight Foundation Scholarship in 2014. His thesis research includes autonomous rotorcraft navigation and control in wind.

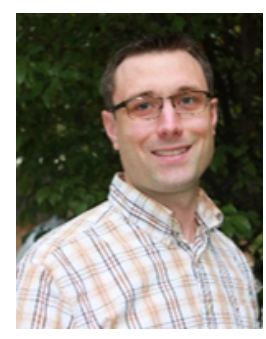

Francis D. Lagor received the B.S. degree in Mechanical Engineering from Villanova University in 2006 and the M.S. degree in Mechanical Engineering and Applied Mechanics from the University of Pennsylvania in 2009. He worked for Lockheed Martin Space Systems Company prior to returning to graduate study in the Department of Aerospace Engineering at the University of Maryland. His research interest is in estimation, navigation and control of robotic systems in complex flow environments.

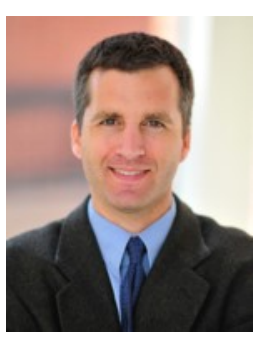

Derek A. Paley received the B.S. degree in Applied Physics from Yale University in 1997 and the Ph.D. degree in Mechanical and Aerospace Engineering from Princeton University in 2007. He received the National Science Foundation CAREER award in 2010 and the Presidential Early Career Award for Scientists and Engineers in 2012. Paley's research interests are in the area of $d y$ namics and control, including cooperative control of autonomous vehicles. 